\title{
Divisors on Division Algebras and Error Correcting Codes
}

\author{
Patrick J. Morandi \\ Department of Mathematical Sciences \\ New Mexico State University \\ Las Cruces, NM 88003 \\ pmorandi@nmsu.edu \\ B.A. Sethuraman* \\ Department of Mathematics \\ California State University, Northridge \\ Northridge, CA 91330 \\ al.sethuraman@csun.edu
}

\section{Introduction}

Recall that a (linear) code is just a $k$-subspace $C$ of $k^{n}$, where $k$ is some finite field. The elements of $C$ are referred to as codewords, and the dimension of the code, $\operatorname{dim}(C)$, is just the dimension of $C$ as a $k$-space. The length of the code is the dimension of the ambient space $k^{n}$; that is, the length of $C$ is $n$. The minimum distance, $d(a, b)$ between two codewords $a=\left(a_{1}, \ldots, a_{n}\right)$ and $b=\left(b_{1}, \ldots, b_{n}\right)$ is defined by $d(a, b)=\left|\left\{i \mid a_{i} \neq b_{i}\right\}\right|$, and the minimum distance of the code, $d(C)$, is defined by $d(C)=\min \{d(a, b) \mid a, b \in C, a \neq b\}$. The weight, $w(a)$, of a codeword $a=\left(a_{1}, \ldots, a_{n}\right)$ is defined by $w(a)=$ $\left|\left\{i \mid a_{i} \neq 0\right\}\right|$. The linearity of the code ensures that $d(C)$ is also equal to $\min \{w(a) \mid a \in C, a \neq 0\}$.

\footnotetext{
*Supported in part by a grant from the N.S.F.
} 
A major goal in coding theory is to construct codes whose minimum distance and dimension are large relative to their length. Goppa Codes were introduced by Goppa in [3] (in a form dual to the one we consider here), and are effective at achieving this goal. One starts with a smooth geometrically irreducible projective curve $X / k$ (and thus, a function field $F / k$ ), and a divisor $E=P_{1}+\cdots+P_{n}$, where the $P_{i}$ are prime divisors of degree 1 . One fixes a divisor $G$ such that the support of $E$ and $G$ are disjoint (this guarantees that any $f \in \mathcal{L}(G)$ is automatically in each valuation ring $\mathcal{O}_{P_{i}}$ ). One then considers the evaluation map ev : $\mathcal{L}(G) \rightarrow k^{n}$ given by $f \mapsto$ $\left(f\left(P_{1}\right), \ldots, f\left(P_{n}\right)\right)$, where by $f\left(P_{i}\right)$, we mean the residue of $f$ with respect to the valuation at $P_{i}$. Since ev is $k$-linear, the image is a $k$-subspace of $k^{n}$; this is the Goppa code associated to the curve $X / k$ and the divisors $E$ and $G$.

We provide in this paper an analogous construction of codes using division algebras $D$ defined over the function field $F / k$. The structure of such a $D$ is of course well-understood from class field theory; we exploit this structure in our construction. Places of the field $F / k$ are now replaced by maximal orders, selected to form a sheaf of maximal orders over $X$. By work of various authors (see [9], [11], and [12]), one has an analogous definition of divisors in this situation, as well as a definition of the spaces $\mathcal{L}(G)$ for any division algebra divisor $G$, and a noncommutative Riemann-Roch Theorem.

Our code is actually an $l$-code, where $l$ is a finite extension of $k$ that arises naturally as the residue of $D$. The function field $l F$ is a maximal subfield of our division algebra; we prove that under certain conditions our $l$-code is the same as the commutative Goppa code obtained by restricting our division algebra divisors in a suitable manner to $l F$.

\section{Maximal Orders and Divisors}

As mentioned in the introduction, we will use maximal orders in our construction of codes. We recall here the basic properties of maximal orders over discrete valuation rings. A good reference for maximal orders is Reiner's book [5]. Let $A$ be an integral domain with quotient field $F$ and let $S$ be an $F$ central simple algebra. A subring $B$ of $S$ with $B \supseteq A$ is an $A$-order if $B$ is a finitely-generated $A$-module and if $B F=S$. If $B$ is maximal with respect to inclusion among all $A$-orders in $S$, then $B$ is said to be a maximal order over $A$. 
If $A$ is a discrete valuation ring with quotient field $F$, then the following properties hold for maximal $A$-orders in an $F$-central simple algebra $S$ :

1. Maximal $A$-orders exist and are unique up to conjugation.

2. If $B$ is a maximal $A$-order in $S$, then the Jacobson radical $J(B)$ is the unique maximal ideal of $B$, and every ideal of $B$ is a power of $J(B)$.

3. There is a $b \in B$ with $J(B)=B b=b B$.

4. The residue ring $\bar{B}=B / J(B)$ is simple and finite dimensional over $\bar{A}=A / J(A)$.

Proofs of these properties can be found in [5, Theorems 18.3, 18.7].

In this paper we need to make use of value functions. If $A$ is a discrete valuation ring and if $B$ is a maximal $A$-order in $S$, then we can define a function $w: S-\{0\} \rightarrow \mathbb{Z}$ in the following way: For $b \in B$, we set $w(b)=n$ if $B b B=J(B)^{n}$. More generally, if $s \in S$, write $s=b \alpha^{-1}$ with $b \in B$ and $\alpha \in A$, and set $w(s)=w(b)-w(\alpha)$. It is not hard to see that $w$ is well defined and that $w$ satisfies the following properties:

1. $w(s+t) \geq \min \{w(s), w(t)\}$;

2. $w(s t) \geq w(s)+w(t)$, and $w(s t)=w(s)+w(t)$ if $s \in Z(S)$;

3. $B=\{s \in S \mid w(s) \geq 0\} \cup\{0\}$ and $J(B)=\{s \in S \mid w(s)>0\} \cup\{0\}$.

Moreover, $w$ is uniquely determined by $B$. We call $w$ the value function on $S$ corresponding to $B$. We will denote by $\Gamma_{B}$ the value group of $B$; that is, $\Gamma_{B}=\operatorname{im}\left(w_{B}\right)=\mathbb{Z}$. We refer the reader to $[6$, Sec. 2$]$ for more information about value functions.

Suppose that $X$ is a smooth geometrically irreducible projective curve defined over a field $k$, and let $F$ be the function field of $X$. Let $S$ be a central simple $F$-algebra, and suppose that we have a maximal $\mathcal{O}_{P}$-order $B_{P}$ in $S$ for each $P \in X$. Let $w_{P}$ be the value function on $S$ corresponding to $B_{P}$. Note that $w_{P}$ depends on both the point $P$ and the choice of maximal order $B_{P}$. Let $e_{P}$ be the index $\left[\mathbb{Z}: w_{P}\left(F^{*}\right)\right]$. We will call $e_{P}$ the ramification index of $S / F$ with respect to $P$.

We will be working with sheaves of maximal orders on $X$. We call a sheaf $\Lambda$ of rings on $X$ a sheaf of maximal orders in $S$ if $\Lambda(U)$ is a maximal 
$\mathcal{O}(U)$-order in $S$ for every proper open subset $U$ of $X$. Let $\mathcal{S}$ be the constant sheaf $\mathcal{S}(U)=S$ on $X$. We recall that any sheaf of maximal orders on $X$ is isomorphic to a subsheaf of $\mathcal{S}$. Here is a sketch of the argument. Let $\zeta$ be the generic point of $X$. If $\Lambda$ is a sheaf of $\mathcal{O}_{X}$-maximal orders in $S$, then the structure maps $\varphi_{U}: \Lambda(U) \rightarrow \Lambda_{\zeta}$ extend by bilinearity to a map $\theta_{U}: \Lambda(U) \otimes_{\mathcal{O}(U)} \mathcal{O}_{\zeta} \rightarrow \Lambda_{\zeta}$. Now $\mathcal{O}_{\zeta}=F$, and for every proper open set $U$, $\Lambda(U)$ is a maximal $\mathcal{O}(U)$ order, so the map $\Lambda(U) \otimes_{\mathcal{O}(U)} F \rightarrow S$ induced by the inclusion $\Lambda(U) \subseteq S$ is an isomorphism. For every proper open set $U$, we thus have a map $\theta_{U}^{\prime}: S \rightarrow \Lambda_{\zeta}$. $\theta_{U}^{\prime}$ is an injection as $S$ is simple. Since any $z \in \Lambda_{\zeta}$ is in $\varphi_{V}(\Lambda(V))$ for some open $V$, we find $z \in \theta_{V}\left(\Lambda(V) \otimes_{\mathcal{O}(V)} \mathcal{O}_{\zeta}\right)$. We may assume $V \subseteq U$. The structure map $\varphi_{U V}: \Lambda(U) \rightarrow \Lambda(V)$ induces an isomorphism $\Lambda(U) \otimes_{\mathcal{O}(U)} \mathcal{O}_{\zeta} \cong \Lambda(V) \otimes_{\mathcal{O}(V)} \mathcal{O}_{\zeta}$ (as each side is isomorphic to $S)$, so we find $z \in \theta_{U}\left(\Lambda(U) \otimes_{\mathcal{O}(U)} \mathcal{O}_{\zeta}\right)$ as well. Thus, $\Lambda_{\zeta} \cong S$. We thus have injective maps $\varphi_{U}: \Lambda(U) \rightarrow \Lambda_{\zeta} \cong S$ for each proper open set $U \subseteq X$. Using the sheaf property of $\Lambda(X)$ and the fact that the structure map $\varphi_{X}: \Lambda(X) \rightarrow \Lambda_{\zeta}$ factors through $\Lambda(U)$ for every proper open set $U$, it is easy to see that $\varphi_{X}$ is injective as well. Since $\varphi_{U}=\varphi_{V} \varphi_{U V}$ for open sets $V \subseteq U$, we have an injective sheaf map from $\Lambda$ to the constant sheaf $\mathcal{S}$.

Lemma 1 Suppose for every $P \in X$ that there is a maximal $\mathcal{O}_{P}$-order $B_{P}$ in $S$, and let $w_{P}$ be the value function on $S$ corresponding to $B_{P}$. Then, for each $s \in S$, we have $w_{P}(s) \geq 0$ for all but finitely many $P$ if and only if there is a sheaf $\Lambda$ of maximal $\mathcal{O}_{X}$-orders on $X$ such that for each $P \in X$, the stalk $\Lambda_{P}$ is equal to $B_{P}$.

Proof: Suppose there is a sheaf $\Lambda \subseteq \mathcal{S}$ of maximal orders such that $\Lambda_{P}=B_{P}$ for each $P \in X$. Let $s \in S$. If $U$ is any proper open subset of $X$, we may write $s=b \alpha^{-1}$ with $b \in \Lambda(U)$ and $\alpha \in \mathcal{O}(U)$. There is a nonempty open subset $V$ of $U$ such that $\alpha^{-1} \in \mathcal{O}(V)$, so $s \in \Lambda(V)$. Thus, for each $P \in V$ we have $w_{P}(s) \geq 0$. Since the complement of $V$ in $X$ is finite, $w_{P}(s) \geq 0$ for all but finitely many $P$.

Conversely, suppose that we have a maximal $\mathcal{O}_{P}$-order $B_{P}$ for each $P \in$ $X$, and that if $w_{P}$ is the value function associated to $B_{P}$, then, for any $s \in S$, we have $w_{P}(s) \geq 0$ for all but finitely many $P$. We define a sheaf $\Lambda$ on $X$ by

$$
\Lambda(U)=\bigcap_{P \in U} B_{P}
$$

for all open subsets $U$ of $X$ with structure maps being the obvious inclusions. It is proved in [7, Ex. 3.4] that $\Lambda(U)$ is a maximal $\mathcal{O}(U)$-order for each proper 
open subset $U$, and so $\Lambda \subseteq \mathcal{S}$ is a sheaf of maximal orders on $X$. Moreover, in [7, Ex. 3.4], it is shown that $\Lambda(U) \mathcal{O}_{P}=B_{P}$ if $P \in U$. This shows that $\Lambda_{P}=B_{P}$ for each $P$.

We now describe the divisor group on $S$ and state a generalization of the Riemann-Roch theorem. If $S$ is a central simple $F$-algebra, let $\Lambda$ be a sheaf of maximal $\mathcal{O}_{X}$-orders on $X$. For each $P$ let $w_{P}$ be the value function on $S$ corresponding to the maximal $\mathcal{O}_{P}$-order $\Lambda_{P}$. The divisor group $\operatorname{div}(S)$ is the free abelian group on the set $\left\{\Lambda_{P}\right\}_{P \in X}$. While this group depends on the choice of sheaf $\Lambda$, we will not deal with more than one sheaf of maximal orders at a time, so we will not need to worry about this dependence. For $E=\sum_{P} n_{P} \Lambda_{P}$, we define $\operatorname{deg}(E)=\sum_{P} n_{P}\left[\bar{\Lambda}_{P}: k\right]$ and $\operatorname{supp}(E)=\left\{\Lambda_{P} \mid n_{P} \neq 0\right\}$. Each $s \in S$ defines a divisor $(s)=\sum_{P} w_{P}(s) \Lambda_{P}$. We point out that this sum is finite by Lemma 1. Unlike the case of fields where principal divisors have degree 0 , we see in the next lemma that the degree of a principal divisor $(s)$ is non-positive, and can be negative. In the proof below we need to work with elements of $\operatorname{div}(F)$ and of $\operatorname{div}(S)$. When we talk about an element $E \in \operatorname{div}(F)$, we will write $\operatorname{deg}_{F}(E)$ for the degree of $E$ as an $F$-divisor.

Lemma 2 Let $s \in S$. Then $\operatorname{deg}((s)) \leq 0$. Moreover, $\operatorname{deg}((s))=0$ if and only if $s \in S^{*}$ and $s \Lambda_{P} s^{-1}=\Lambda_{P}$ for every $P \in X$.

Proof: Let $v_{P}$ be the normalized valuation on $F$ with valuation ring $\mathcal{O}_{P}$. Then $\left.w_{P}\right|_{F}=e_{P} v_{P}$, where $e_{P}$ is the ramification index at $P$ of $S$ over $F$. Moreover, by [6, Prop. 2.6], if $n=\operatorname{deg}(S)$, then $w_{P}(s) \leq n^{-1} w_{P}(\operatorname{Nrd}(s))$. With these facts in mind, we see that

$$
\begin{aligned}
\operatorname{deg}((s)) & =\sum_{P} w_{P}(s)\left[\overline{\Lambda_{P}}: k\right] \\
& =\sum_{P} w_{P}(s)\left[\overline{\Lambda_{P}}: \overline{\mathcal{O}_{P}}\right] \cdot\left[\overline{\mathcal{O}_{P}}: k\right] \\
& \leq \sum_{P} \frac{1}{n} w_{P}(\operatorname{Nrd}(s))\left[\overline{\Lambda_{P}}: \overline{\mathcal{O}_{P}}\right] \operatorname{deg}_{F}(P) \\
& =\sum_{P} \frac{1}{n} e_{P}\left[\overline{\Lambda_{P}}: \overline{\mathcal{O}_{P}}\right] v_{P}(\operatorname{Nrd}(s)) \operatorname{deg}_{F}(P) \\
& =\sum_{P} n v_{P}(\operatorname{Nrd}(s)) \operatorname{deg}_{F}(P)=n \operatorname{deg}_{F}((\operatorname{Nrd}(s))) \\
& =0 .
\end{aligned}
$$


The second to last equality holds since $[S: F]=e_{P}\left[\overline{\Lambda_{P}}: \overline{\mathcal{O}_{P}}\right]$ for all $P \in X$ by [5, Theorems 13.3, 18.2, Corollary 17.5].

For the second statement, we note that the inequalities above show that $\operatorname{deg}((s))<0$ if and only if $w_{P}(s)<n^{-1} w_{P}(\operatorname{Nrd}(s))$ for some $P$. However, if $s \in S^{*}$, then $w_{P}(s)=n^{-1} w_{P}(\operatorname{Nrd}(s))$ if and only if $s \Lambda_{P} s^{-1}=\Lambda_{P}$ by [6, Prop. 2.6]. Therefore, if $s \in S^{*}$, then $\operatorname{deg}((s))=0$ if and only if $s \Lambda_{P} s^{-1}=\Lambda_{P}$ for every $P \in X$. To finish the argument, given that $\operatorname{deg}((s))=0$, we show that $s$ must be in $S^{*}$. Pick a point $P \in X$, and set $J\left(\Lambda_{P}\right)=\pi_{P} \Lambda_{P}$, where $\pi_{P} \Lambda_{P} \pi_{P}^{-1}=\Lambda_{P}$. We have $s=\pi_{P}^{n} u$ for some $u$ with $w_{P}(u)=0$, and where $n=w_{P}(s)$. If $w_{P}(s)=n^{-1} w_{P}(\operatorname{Nrd}(s))$, then $0=w_{P}(\operatorname{Nrd}(u))$, which forces $u \in \Lambda_{P}^{*} \subseteq S^{*}$ by the argument immediately preceding [6, Prop. 2.6]. Therefore, $s=\pi_{P}^{n} u \in S^{*}$.

Example. Let $p$ be an odd prime, let $k$ be a finite field of characteristic $p$, let $a \in k^{*}-k^{* 2}$, and let $D$ be the quaternion algebra

$$
D=\left(\frac{a, t}{k(t)}\right) \text {. }
$$

Let $1, i, j, k$ be the standard quaternion basis for $D$. The field $k(t)$ is the function field of $\mathbb{P}^{1}$, so points are in 1-1 correspondence with irreducible polynomials over $k$, except for the point $P_{\infty}$ at infinity which corresponds to the dvr $k\left[t^{-1}\right]_{\left(t^{-1}\right)}$. If a point $P$ corresponds to $p(t) \neq t$, then we can choose $\Lambda_{P}=\left(\frac{a, t}{\mathcal{O}_{P}}\right)$. Let $P_{0}$ be the point corresponding to $t$. There are unique maximal orders over $\mathcal{O}_{P_{0}}$ and $\mathcal{O}_{P_{\infty}}$, which can be shown to be $\left(\frac{a, t}{\mathcal{O}_{P_{0}}}\right)$ and $\left(\frac{a, t^{-1}}{\mathcal{O}_{P_{\infty}}}\right)$, respectively. Construct a sheaf $\Lambda$ as in the proof of Lemma 1. (The condition $w_{P}(d) \geq 0$ almost everywhere is easily verified: every $d \in D^{*}$ is of the form $b / \alpha$ for some $b \in\left(\frac{a, t}{k[t]}\right)$ and some $\alpha \in k[t]-\{0\}$. Then $d \in \Lambda_{P}$ for all $P$ such that $P \neq P_{\infty}$ and $\alpha^{-1} \in \mathcal{O}_{P}$.) With this sheaf, we verify that the divisor of $1+j$ is equal to $-P_{\infty}$, so $(1+j)$ has negative degree. By Proposition 9 ahead (with $x=j$ ), we find that 1 and $j$ form a strongly orthogonal basis for $D / k(t)(\sqrt{a})$, so $w_{P}(1+j)=\min \left(w_{P}(1), w_{P}(j)\right)$ for all $P \in X$. Since $t$ is a unit in $\mathcal{O}_{P}$ for all $P$ except those that correspond to $t$ or $1 / t$, and since $j^{-1}=t^{-1} j$, we find that $j$ conjugates $\Lambda_{P}$ to itself for all such $P$. By [6, Prop. 2.6], $w_{P}(j)=0$ for all such $P$. As for $P_{0}$ and $P_{\infty}$, the maximal orders at these points are valuation rings, and $w_{P_{0}}(j)=1$, and $w_{P_{\infty}}(j)=-1$. Thus, $(1+j)=-P_{\infty}$ as claimed. 
We define a map $\phi_{S}: \operatorname{div}(F) \rightarrow \operatorname{div}(S)$ by $\phi_{S}\left(\sum_{P} n_{P} P\right)=\sum_{P}\left(n_{P} e_{P} \Lambda_{P}\right)$. The main property of this map that we need is given in the following lemma.

Lemma 3 If $E \in \operatorname{div}(F)$, then $\operatorname{deg}\left(\phi_{S}(E)\right)=[S: F] \operatorname{deg}_{F}(E)$.

Proof: If $E=\sum_{P} n_{P} P$, then we have

$$
\begin{aligned}
\operatorname{deg}\left(\phi_{S}(E)\right) & =\sum_{P} n_{P} e_{P} \operatorname{deg}\left(\Lambda_{P}\right)=\sum_{P} n_{P} e_{P}\left[\overline{\Lambda_{P}}: k\right] \\
& =\sum_{P} n_{P} e_{P}\left[\overline{\Lambda_{P}}: \overline{\mathcal{O}_{P}}\right]\left[\overline{\mathcal{O}_{P}}: k\right] \\
& =\sum_{P} n_{P}[S: F] \operatorname{deg}(P)=[S: F] \operatorname{deg}(E) .
\end{aligned}
$$

If $E, E^{\prime}$ are divisors on $S$, we write $E \geq E^{\prime}$ if $E-E^{\prime}=\sum n_{P} \Lambda_{P}$ with each $n_{P} \geq 0$. Let $\mathcal{L}(E)=\{s \in S \mid(s)+E \geq 0\}$, a $k$-subspace of $S$. As a consequence of Lemma 2, we point out that if $\operatorname{deg}(E)<0$, then $\mathcal{L}(E)=0$. To see this, if $f \in \mathcal{L}(E)$ is nonzero, then $(f)+E \geq 0$, so $\operatorname{deg}((f)+E)=$ $\operatorname{deg}((f))+\operatorname{deg}(E) \leq \operatorname{deg}(E)<0$, a contradiction to the condition $(f)+E \geq 0$. Let $\mathcal{C}$ be a canonical divisor on $F$, and set $\mathcal{K}=\phi_{S}(\mathcal{C})+\sum_{P}\left(e_{P}-1\right) \Lambda_{P} \in$ $\operatorname{div}(S)$. We now state the generalization of the Riemann-Roch theorem to this setting.

Theorem 4 (Riemann-Roch) Let $g=\max _{E \in \operatorname{div}(S)}\{\operatorname{deg}(E)+1-\operatorname{dim}(\mathcal{L}(E))\}$. Then $g$ exists, and for all divisors $E$, we have $\operatorname{dim}(\mathcal{L}(E))=\operatorname{deg}(E)+1-$ $g+\operatorname{dim}(\mathcal{K}-E)$.

A proof of this theorem can be found in [12, Satz 12] or [11, Theorem 3.17]. We would like to thank J.-L Colliot-Thélène for telling us about Witt's paper. Analogous to the commutative case, we have $\operatorname{deg}(\mathcal{K})=2 g-2$. By the definition of $\mathcal{K}$ and Lemma 3 , if $g_{F}$ is the genus of $F$, we see that

$$
g=[S: F]\left(g_{F}-1\right)+1+\frac{1}{2} \sum_{P}\left(e_{P}-1\right) \operatorname{deg}\left(\Lambda_{P}\right)
$$

In the next section, in order to construct sheaves of maximal orders, we will use the fact that if $U$ is a proper open subset of $X$, then $\bigcap_{P \in U} \mathcal{O}_{P}$ is a Dedekind domain of $F$. This is a standard result, but we give a proof here for the convenience of the reader. 
Lemma 5 If $U$ is a proper open subset of $X$, then $\bigcap_{P \in U} \mathcal{O}_{P}$ is a Dedekind domain of $F$.

Proof: Let $X-U=\left\{Q_{1}, \ldots, Q_{n}\right\}$, let $E=\sum_{i} n_{i} Q_{i} \in \operatorname{div}(F)$, and let $E_{i}=E-Q_{i}$, where the $n_{i}$ are positive integers. If we choose the $n_{i}$ large enough, then by the Riemann-Roch theorem, $\operatorname{dim}(\mathcal{L}(E))$ is larger than each $\operatorname{dim}\left(\mathcal{L}\left(E_{i}\right)\right)$ since $\operatorname{deg}(E)>\operatorname{deg}\left(E_{i}\right)$ for each $i$. Each of these are finitedimensional $k$-vector spaces, so $\mathcal{L}(E)$ properly contains the union $\bigcup_{i} \mathcal{L}\left(E_{i}\right)$. Choose $x \in \mathcal{L}(E)$ such that $x \notin \mathcal{L}\left(E_{i}\right)$ for each $i$. Then $(x)+E \geq 0$ but $(x)+E_{i} \nsupseteq 0$ for each $i$. This forces $v_{P_{i}}(x)=-n_{i}$ for each $i$, and $v_{P}(x) \geq 0$ for each $P \in U$. Consequently, the valuation rings of $F$ that contain $x$ are precisely the $\mathcal{O}_{P}$ for $P \in U$. Note that $x \notin k$, so $F$ is algebraic over $k(x)$. A valuation ring of $F / k$ contains $x$ if and only if it contains $k[x]$, so we see that the integral closure of $k[x]$ in $F$ is $\bigcap_{P \in U} \mathcal{O}_{P}$. However, since $k[x]$ is a Dedekind domain of $k(x)$, the ring $\bigcap_{P \in U} \mathcal{O}_{P}$ is a Dedekind domain of $F$ by [5, Theorem 4.4].

\section{The Construction of the Code}

Let $F / k$ be a function field in one variable over the finite field $k$. One has the following sequence from class field theory (see the discussion in $[5$, pages 277-278] for instance)

$$
0 \longrightarrow \operatorname{Br}(F) \longrightarrow \oplus_{P} \operatorname{Br}\left(F_{P}\right) \longrightarrow \mathbb{Q} / \mathbb{Z} \longrightarrow 0,
$$

where the sum in the middle term is over all the places of the field $F$. For any $D \in \operatorname{Br}(F), D \otimes_{F} F_{P}$ is trivial almost everywhere; thus the second map in the sequence above, which is simply the sum of the various restriction maps, is well-defined. It is well-known that for each $P$, there is a canonical map Inv $: \operatorname{Br}\left(F_{P}\right) \rightarrow \mathbb{Q} / \mathbb{Z}$ which is an isomorphism, and the third map above is just the sum of the Inv maps for each $P$. The map Inv can be explicitly described as follows: any division algebra $D / F_{P}$ of degree $r$ is isomorphic to the cyclic algebra $\left(W / F_{P}, \sigma, \pi^{i}\right)$, where $W$ is the unique unramified field extension of $F_{P}$ of degree $r, \pi$ is a uniformizer for $F_{P}, \sigma$ is a generator of the Galois group of the cyclic field extension $W / F_{P}$ chosen so as to induce the Frobenius automorphism at the residue level, and $1 \leq i<r$ with $\operatorname{gcd}(i, r)=$ 1. With this isomorphism at hand, $\operatorname{Inv}(D)$ is defined to be $i / r$. (See the 
discussion in [5, pages 263-266] for the description of division algebras over local fields.)

$D$ is said to be ramified at a place $P$ if $D \otimes_{F} F_{P}$ is not split. Moreover, if $\operatorname{Inv}\left(D \otimes_{F} F_{P}\right)=i / s$ with $\operatorname{gcd}(i, s)=1$, then $s$ is said to be the local index of $D$ at $P$, and ind $(D)$ is simply the least common multiple of the various local indices (see [5, Theorem 32.19]).

Now let $X / k$ be a smooth geometrically irreducible projective curve, and $F / k$ the associated function field. Let $P_{1}, \ldots, P_{n}$ be places of $F / k$ of degree 1. Choose integers $r, j_{1}, \ldots, j_{n}$ such that $1 \leq j_{i}<r, \operatorname{gcd}\left(j_{i}, r\right)=1$, and $\sum_{i}\left(j_{i} / r\right)=0$ in $\mathbb{Q} / \mathbb{Z}$. Thus, the exact sequence $(1)$ shows that there is a division algebra $D / F$ ramified at exactly the places $P_{i}$, and at each $P_{i}, D$ has local index $r$. It follows that ind $(D)=r$ as well. Also, the residue field of $F_{P_{i}}$ is just $k$ since the $P_{i}$ have degree 1 , and if $l / k$ is the unique extension of $k$ of degree $r$, then $l F_{P_{i}}$ is the unique unramified extension of $F_{P_{i}}$ of degree $r$. Thus, by the discussion in the previous paragraph, $D \otimes_{F} F_{P_{i}}$ is isomorphic to $\left(l F_{P_{i}} / F_{P_{i}}, \sigma_{i}, \pi^{j_{i}}\right)$, where $\sigma_{i}$ is a generator of $\operatorname{Gal}\left(l F_{P_{i}} / F_{P_{i}}\right)$ chosen so as to induce the Frobenius automorphism on $l / k$.

We first show that $L=l F$ is a maximal subfield of $D$. For any place $Q$ of $L$, let $P$ be the corresponding place of $F$ induced by $Q$. The completion of $L$ at $Q$ is isomorphic to the compositum $l F_{P}$. If $P$ is not one of the selected $P_{i}$, then $D \otimes_{F} F_{P}$ is already split, so $\left(D \otimes_{F} L\right) \otimes_{L} L_{Q}=\left(D \otimes_{F} F_{P}\right) \otimes_{F_{P}} l F_{P}$ is split. If $P$ is one of the selected $P_{i}$, then, as remarked in the last paragraph, $l F_{P}$ is a maximal subfield of $D \otimes_{F} F_{P}$, so once again, $\left(D \otimes_{F} L\right) \otimes_{L} L_{Q}=$ $\left(D \otimes_{F} F_{P}\right) \otimes_{F_{P}} l F_{P}$ is split. It follows that $D \otimes_{F} L$ is split everywhere, so by our exact sequence $(1), L$ splits $D$. Since $[L: F]=r$ (as $k$ is algebraically closed in $F$, this follows from the geometric irreducibility of $X / k), L$ is indeed a maximal subfield of $D / F$.

We construct a sheaf of maximal orders $\Lambda$ on our curve $X$ as follows: Let $A=\bigcap \mathcal{O}_{Q}$, where the intersection runs across all places $Q$ of $F$ except the chosen places $P_{i}$. As described in Lemma $5, A$ is a Dedekind domain. Since $l \subset D, l A$ is a free finitely generated $A$ module. Choose a basis $\left\{b_{1}=1, b_{2}, \ldots, b_{r}\right\}$ of $D / L$, and consider the free $A$ module $M=\bigoplus_{i=1}^{n}(l A) b_{i}$. Then $M F=D$, and $l A \subseteq O_{l}(M)$, where $O_{l}(M)=\{x \in D \mid x M \subseteq M\}$. As discussed in [5, page 109], $O_{l}(M)$ is an $A$-order in $D$, and is hence contained in a maximal $A$-order; call this maximal order $B$. Then, for each place $Q$ of $F$ $\left(Q \notin\left\{P_{1}, \ldots, P_{n}\right\}\right), B \otimes_{A} \mathcal{O}_{Q}$ is a maximal $\mathcal{O}_{Q}$ order. We set $\Lambda_{Q}=B \otimes_{A} \mathcal{O}_{Q}$.

As for the places $P_{1}, \ldots, P_{n}$, there is a unique maximal order over each $\mathcal{O}_{P_{i}}$ which is in fact a valuation ring. (This follows, for instance, from $[5$, 
Theorems 12.8 and 11.5]. We set $\Lambda_{P_{i}}$ to be this maximal $\mathcal{O}_{P_{i}}$ order.

Now let $w_{Q}$ be the value function associated with this choice of maximal orders and consider the sheaf of rings $\Lambda$ given by the assignment $U \mapsto$ $\bigcap_{Q \in U} \Lambda_{Q}$. Exactly as in the argument in the proof of Lemma 1 , any $s \in D$ can be written as $b \alpha^{-1}$ for some $b \in \Lambda(U)$, where $U=X-\left\{P_{1}, \ldots, P_{n}\right\}$, and for all but finitely many points $Q \in U, \alpha^{-1} \in \Lambda_{Q}$. It follows that $w_{Q}(s) \geq 0$ for all but finitely many points $Q \in U$. Since the complement of $U$ contains only finitely many points, Lemma 1 shows that $\Lambda$ is indeed a sheaf of maximal orders, whose stalk at each point $Q \in X$ is $\Lambda_{Q}$.

We let $\mathcal{P}$ be the $D$-divisor $\Lambda_{P_{1}}+\cdots+\Lambda_{P_{n}}$, and we let $G$ be any $D$-divisor whose support is disjoint from the $P_{i}$, and such that $\mathcal{L}(G) \neq\{0\}$. The condition on the supports guarantees that any $f \in \mathcal{L}(G)$ is automatically in each $\Lambda_{P_{i}}$. Since each $\Lambda_{P_{i}}$ is just the valuation ring corresponding to the $P_{i^{-}}$ adic valuation, the factor ring $\Lambda_{P_{i}} / J\left(\Lambda_{P_{i}}\right)$, where $J\left(\Lambda_{P_{i}}\right)$ is the maximal ideal of $\Lambda_{P_{i}}$, is just the residue field of $D$ with respect to the $P_{i}$-adic valuation. The residue of $D$ is the same as the residue of the completion $D \otimes_{F} F_{P_{i}}$, which is just $l$. We thus have, exactly as in the commutative case, a map

$$
\begin{aligned}
& \text { ev }: \mathcal{L}(G) \longrightarrow l^{n} \\
& f \quad \mapsto \quad\left(f\left(P_{1}\right), \ldots, f\left(P_{n}\right)\right),
\end{aligned}
$$

where we have written $f\left(P_{i}\right)$ for the residue of $f$ modulo the maximal ideal $J\left(\Lambda_{P_{i}}\right)$ of $\Lambda_{P_{i}}$.

By our choice of maximal orders above, the field $l$ is contained in every maximal order $\Lambda_{Q}$ for $Q \notin\left\{P_{1}, \ldots, P_{n}\right\}$. Since each $\Lambda_{P_{i}}$ is a valuation ring, and since any valuation on $l$ must be trivial, $l$ must be contained in each $\Lambda_{P_{i}}$ as well. It follows that for any $Q, w_{Q}(x) \geq 0$ for all $x \in l^{*}$. Hence, for any $Q$, any $x \in l^{*}$, and any $f \in \mathcal{L}(G)$,

$$
w_{Q}(x f) \geq w_{Q}(x)+w_{Q}(f) \geq w_{Q}(f) \geq-w_{Q}(G),
$$

so $x f \in \mathcal{L}(G)$. Thus, besides being a $k$-space, $\mathcal{L}(G)$ is also an $l$-space. Moreover, $x\left(P_{i}\right)$ is just $x$ for any $P_{i}$, as the $P_{i}$-adic valuation is trivial on $l$. It follows from this that the map ev above is an $l$-linear map. (In fact, each $x \in l^{*}$ conjugates each $\Lambda_{Q}$ to itself, so by [6, Prop. 2.6 and Lemma 2.2], $w_{Q}(x)=0$, and $\left.w_{Q}(x f)=w_{Q}(x)+w_{Q}(f)=w_{Q}(f).\right)$

The image of ev is thus an $l$-subspace of $l^{n}$; this will be our noncommutative Goppa code associated to $D / F$ and the divisors $\mathcal{P}$ and $G$. We denote it $C_{D}(\mathcal{P}, G)$. 
We point out that while different choices in our sheaf construction may lead to different (e.g., non-isomorphic) sheaves, the ring of constants $\bigcap_{Q \in X} \Lambda_{Q}$ is uniquely determined up to isomorphism by the division algebra $D$ since $k$ is finite, by a theorem of Schofield [10, Theorem 1.1]. We are working with a specific choice of sheaf to see that $l$ is a subring of the ring of constants. In fact, it is not hard to see that $l=\bigcap_{Q \in X} \Lambda_{Q}$, so $l$ is the full ring of constants of the sheaf $\Lambda$.

We determine in the next proposition the minimum distance and the dimension of the code $C_{D}(\mathcal{P}, G)$. The methods are analogous to those used for commutative Goppa codes (see [3]). Note that since $\overline{\Lambda_{P_{i}}}=l$ for each $i$, we have $\operatorname{deg}\left(\Lambda_{P_{i}}\right)=r=[l: k]$. Therefore, $\operatorname{deg}(\mathcal{P})=r n$.

Proposition $6 \operatorname{dim}\left(C_{D}(\mathcal{P}, G)\right)=\operatorname{dim}(\mathcal{L}(G))-\operatorname{dim}(\mathcal{L}(G-\mathcal{P}))$, and $d\left(C_{D}(\mathcal{P}, G)\right) \geq$ $n-r^{-1} \operatorname{deg}(G)$. Moreover, if $\operatorname{deg}(G)<r n$, then $\left.\operatorname{dim}\left(C_{D}(\mathcal{P}, G)\right)=\operatorname{dim} \mathcal{L}(G)\right) \geq$ $\operatorname{deg}(G)+1-g$, where $g$ is the genus of $D$.

Proof: The kernel of ev is $\left\{f \in \mathcal{L}(G) \mid v_{P_{i}}(f) \geq 1, i=1, \ldots, n\right\}$, which is precisely $\mathcal{L}(G-\mathcal{P})$. The result on the dimension immediately follows. As for the distance, write $d$ for $d\left(C_{D}(\mathcal{P}, G)\right)$ and let $f \in \mathcal{L}(G), f \neq 0$, be such that $w(\operatorname{ev}(f))=d$. Then $f$ is in the maximal ideal of exactly $n-d$ of the $\Lambda_{P_{i}}$, say $\Lambda_{P_{i_{1}}}, \ldots, \Lambda_{P_{i_{n-d}}}$. It follows that $f \in \mathcal{L}\left(G-\left(\Lambda_{P_{i_{1}}}+\cdots+\Lambda_{P_{i_{n-d}}}\right)\right)$. Hence, $\mathcal{L}\left(G-\left(\Lambda_{P_{i_{1}}}+\cdots+\Lambda_{P_{i_{n-d}}}\right)\right) \neq\{0\}$. It follows that $\operatorname{deg}\left(G-\left(\Lambda_{P_{i_{1}}}+\cdots+\right.\right.$ $\left.\left.\Lambda_{P_{i_{n-d}}}\right)\right) \geq 0$, since $\mathcal{L}(H)=\{0\}$ for a divisor $H$ of negative degree. Thus, $\operatorname{deg}(G)-r(n-d) \geq 0$, or $d \geq n-r^{-1} \operatorname{deg}(G)$.

For the second statement, if $\operatorname{deg}(G)<r n=\operatorname{deg}(\mathcal{P})$, then $\operatorname{deg}(G-\mathcal{P})<$ 0 , so $\mathcal{L}(G-\mathcal{P})=\{0\}$. It follows from the paragraph above that ev is then an injective map. Moreover, by the Riemann-Roch theorem, we have $\operatorname{dim}\left(C_{D}(\mathcal{P}, G)\right)=\operatorname{dim}(\mathcal{L}(G)) \geq \operatorname{deg}(G)+1-g$.

\section{Relation between $C_{D}(\mathcal{P}, G)$ and $C_{L}(\psi(\mathcal{P}), \psi(G))$}

We continue to use the notation of the previous sections: $P_{1}, \ldots, P_{n}$ are rational points on $X$, and $D$ is a division algebra with center $F$, the function field of $X / k$, such that the local index of $D$ at each $P_{i}$ is equal to the index $r$ of $D$, and the local index of $D$ at any point $P \neq P_{i}$ is 1 . Recall that we have a sheaf $\Lambda$ of maximal orders on $D$, that $\Lambda$ is a subsheaf of the constant sheaf $U \mapsto D$, and that each stalk $\Lambda_{P}$ contains $l$. Note that since 
$l / k$ is a cyclic Galois extension and $l, F$ are linearly disjoint over $k$, the extension $L / F$ is also cyclic Galois. Let $\sigma$ be a generator of $\operatorname{Gal}(L / F)$. Since $L$ is a maximal subfield of $D$, we may write $D$ as a cyclic crossed product $D=(L / F, \sigma, a)=\bigoplus_{i=0}^{r-1} L x^{i}$, where $x^{r}=a \in F$ and $x b x^{-1}=\sigma(b)$ for all $b \in L$. We will make use of this description of $D$ in a number of places below.

If $K$ is a field extension of $F$, recall the canonical map $\psi_{K}: \operatorname{div}(F) \rightarrow$ $\operatorname{div}(K)$ that satisfies $\psi_{K}(P)=\sum e_{Q / P} Q$, where the sum is over all $K$-points $Q$ lying over $P$, and where $e_{Q / P}$ is the ramification index of $Q$ over $P$. In terms of valuations, $e_{Q / P}$ is the ramification index of $K / F$ relative to the valuation ring of $K$ corresponding to $Q$. We will refer to this valuation ring by $\mathcal{O}_{K, Q}$. For our maximal subfield $l F=L$ of $D$, we write $\psi$ for $\psi_{L}$. We also view the map $\psi$ as a map from $\operatorname{div}(D)$ to $\operatorname{div}(L)$ by sending $\Lambda_{P}$ to $\psi(P)$. Since $L / F$ is everywhere unramified, $e_{Q / P}=1$ for every $P$ and every $Q$ lying over $P$, and, for each of the points $P_{i}$, there is a unique point $Q_{i}$ lying over $P_{i}$. This follows from the fundamental equality $[L: F]=\sum_{Q / P} e_{Q / P} f_{Q / P}$, where $f_{Q / P}=\left[\overline{\mathcal{O}_{L, Q}}: \overline{\mathcal{O}_{P}}\right]$, and from the fact that the residue field of any point extending $P_{i}$ is $l$, an extension of $k=\overline{\mathcal{O}_{P_{i}}}$ of degree $[L: F]$. As above, let $\mathcal{P}=\sum_{i} \Lambda_{P_{i}}$ and let $G$ be a divisor with $\operatorname{supp}(G) \cap\left\{\Lambda_{P_{1}}, \ldots, \Lambda_{P_{n}}\right\}=\varnothing$. In the next lemma we compare our code with a Goppa code constructed from the algebraic function field $L / l$. To refer to such a Goppa code we denote it by $C_{L}\left(E^{\prime}, G^{\prime}\right)$ if $E^{\prime}, G^{\prime}$ are appropriately chosen divisors on $L$.

Lemma 7 The space $\mathcal{L}(\psi(G))$ is canonically isomorphic to $\mathcal{L}(G) \cap L$, and, with the identification of $\mathcal{L}(\psi(G))$ as a subspace of $\mathcal{L}(G)$, the image $\operatorname{ev}(\mathcal{L}(\psi(G)))$ of $\mathcal{L}(\psi(G))$ under the evaluation map ev : $\mathcal{L}(G) \rightarrow l^{n}$ is the Goppa code $C_{L}(\psi(\mathcal{P}), \psi(G))$.

Proof: Let $f \in L$ satisfy $(f)+\psi(G) \geq 0$ in $\operatorname{div}(L)$. Given $P \in$ $X-\left\{P_{1}, \ldots, P_{n}\right\}$, let $Q_{1}, \ldots, Q_{g}$ be the extensions of $P$ to $L$. Let $n_{P}$ be the coefficient of $\Lambda_{P}$ in $G$. Thus, $n_{P}$ is the coefficient of each $Q_{i}$ in $\psi(G)$. Consequently, $v_{Q_{i}}(f)+n_{P} \geq 0$ for each $i$. Let $\pi$ be a uniformizer for $\mathcal{O}_{P}$. Then $\pi$ is a uniformizer for each $Q_{i}$ since $L / F$ is everywhere unramified. So, $v_{Q_{i}}\left(\pi^{n_{P}} f\right) \geq 0$ for all $i$, so $\pi^{n_{P}} f$ lies in all of the valuation rings that extend $\mathcal{O}_{P}$. But this means that $\pi^{n_{P}} f$ is in the integral closure of $\mathcal{O}_{P}$ in $L$, which is $l \mathcal{O}_{P}$. (To see this, if $B$ is the integral closure of $\mathcal{O}_{P}$ in $L$, take a basis $l_{1}, \ldots, l_{r}$ of $l / k$, and let $l_{1}^{\prime}, \ldots, l_{r}^{\prime}$ be the dual basis relative to $\operatorname{Tr}_{l / k}$. Then $l_{1}^{\prime}, \ldots, l_{r}^{\prime}$ is also an $F$-basis of $L$. Suppose that $b \in B$, and write $b=\sum_{j} l_{j}^{\prime} a_{j}$ with $a_{j} \in F$. Then $b l_{i}=\sum_{j} l_{i} l_{j}^{\prime} a_{j}$, so $\operatorname{Tr}_{L / F}\left(b l_{i}\right)=\sum_{j} \operatorname{Tr}_{l / k}\left(l_{i} l_{j}^{\prime}\right) a_{j}=a_{i}$. 
But, $\operatorname{Tr}_{L / F}(B) \subseteq \mathcal{O}_{P}$, so each $a_{i} \in \mathcal{O}_{P}$. Thus, $B=\sum l_{j}^{\prime} \mathcal{O}_{P}=l \mathcal{O}_{P}$.) Hence, $\pi^{n_{P}} f \in \Lambda_{P}$. Since $\pi$ is also a uniformizer for $\Lambda_{P}$ and is in its center, we have $w_{P}\left(\pi^{n_{P}} f\right)=w_{P}(f)+n_{P} \geq 0$, so $f \in \mathcal{L}(G)$. For $P \in\left\{P_{1}, \ldots, P_{n}\right\}$, $l \mathcal{O}_{P_{i}}$ is the unique valuation ring extending $\mathcal{O}_{P_{i}}$, and if say $v_{i}^{\prime}$ denotes this valuation on $L$, then $(f)+\psi(G) \geq 0$ implies that $v_{i}^{\prime}(f) \geq 0$ for each $i$, which implies that $f \in \Lambda_{P_{i}}$ for each $i$. Thus, $w_{P_{i}}(f) \geq 0$ for each $i$. Therefore, $\mathcal{L}(\psi(G)) \subseteq \mathcal{L}(G) \cap L$.

For the reverse inclusion, take $f \in \mathcal{L}(G) \cap L$. Then, for $P \in X-$ $\left\{P_{1}, \ldots, P_{n}\right\}, w_{P}(f)+n_{P} \geq 0$. If $\pi \in \mathcal{O}_{P}$ is a uniformizer for $\mathcal{O}_{P}$ and $\Lambda_{P}$, then $f=\pi^{-n_{P}} u$ for some $u \in \Lambda_{P}$. But this puts $u$ in $\Lambda_{P} \cap L$, which is integral over $\mathcal{O}_{P}$. Thus, $u$ is in every valuation ring of $L$ extending $\mathcal{O}_{P}$; in other words, $v_{Q}(f) \geq-n_{P}$ for every valuation ring of $L$ extending $\mathcal{O}_{P}$. For $P \in\left\{P_{1}, \ldots, P_{n}\right\}$, we have $w_{P_{i}}(f) \geq 0$, so $f \in \Lambda_{P_{i}} \cap L$. As before, $\Lambda_{P_{i}}$ is a valuation ring, so $\Lambda_{P_{i}} \cap L$ is the (unique) valuation ring of $L$ that extends $\mathcal{O}_{P_{i}}$. Thus, $v_{P_{i}}(f) \geq 0$ for each $i$. Thus, we have proven $\mathcal{L}(\psi(G))=\mathcal{L}(G) \cap L$.

Consider ev : $\mathcal{L}(G) \rightarrow l^{n}$, where $\operatorname{ev}(f)=\left(f\left(P_{1}\right), \ldots, f\left(P_{n}\right)\right)$. If $f \in$ $\mathcal{L}(\psi(G))$, we claim that $f\left(Q_{i}\right)=f\left(P_{i}\right)$ for each $i$, and so $\operatorname{ev}(\mathcal{L}(\psi(G)))=$ $\left\{\left(f\left(Q_{1}\right), \ldots, f\left(Q_{n}\right)\right) \mid f \in \mathcal{L}(\psi(G))\right\}$. This is $C_{L}(\psi(\mathcal{P}), \psi(G))$, finishing the proof of the lemma once we prove the claim. To do this, note that if $\mathfrak{m}_{L, Q_{i}}$ is the maximal ideal of $\mathcal{O}_{L, Q_{i}}$, then $f\left(Q_{i}\right)=f+\mathfrak{m}_{L, Q_{i}} \in \overline{\mathcal{O}_{L, Q_{i}}}=l=\overline{\Lambda_{P_{i}}}$. Also, viewing $f \in D$, we have $f\left(P_{i}\right)=f+J\left(\Lambda_{P_{i}}\right)$. But, $\Lambda_{P_{i}}$ contains $\mathcal{O}_{L, Q_{i}}$ and $J\left(\Lambda_{P_{i}}\right) \cap \mathcal{O}_{L, Q_{i}}=\mathfrak{m}_{L, Q_{i}}$, so $f+\mathfrak{m}_{L, Q_{i}}=f+J\left(\Lambda_{P_{i}}\right)$. In other words, $f\left(Q_{i}\right)=f\left(P_{i}\right)$.

We say that an $L$-basis $d_{1}, \ldots, d_{r}$ of $D$ is an orthogonal basis with respect to a maximal order $\Lambda_{P} \in X$ with associated value function $w_{P}$, provided that $w_{P}\left(\sum_{i} l_{i} d_{i}\right)=\min _{i}\left\{w_{P}\left(l_{i} d_{i}\right)\right\}$ for all $l_{i} \in L$. If, in addition, $w_{P}\left(\sum_{i} l_{i} d_{i}\right)=\min _{i}\left\{w_{P}\left(l_{i}\right)+w_{P}\left(d_{i}\right)\right\}$, we will call the $L$-basis $d_{1}, \ldots, d_{r}$ a strongly orthogonal basis with respect to $\Lambda_{P}$.

Lemma 8 With $D=\bigoplus_{i=0}^{r-1} L x^{i}$ as in the beginning of this section,

1. If $D$ is split at $P$, let $d_{1}, \ldots, d_{r}$ be units in $\Lambda_{P}$ such that $\overline{d_{1}}, \ldots, \overline{d_{r}}$ is a basis for $\overline{\Lambda_{P}}$ over $\overline{\Lambda_{P} \cap L}$. Then $d_{1}, \ldots, d_{r}$ is a strongly orthogonal basis for $D$ with respect to $\Lambda_{P}$.

2. If $P=P_{i}$, then $1, x, \ldots, x^{r-1}$ is a strongly orthogonal basis for $D$ with respect to $\Lambda_{P}$.

Proof: Suppose that $d_{1}, \ldots, d_{r}$ are units in $\Lambda_{P}$ such that $\overline{d_{1}}, \ldots, \overline{d_{r}}$ is a basis for $\overline{\Lambda_{P}}$ over $\overline{\Lambda_{P} \cap L}$. Note that $w_{P}\left(d_{i}\right)=0$ for each $i$, and that since 
$d_{i} \in \Lambda_{P}^{*}$, we have $w_{P}\left(e d_{i}\right)=w_{P}(e)$ for all $e \in D$ by the definition of the value function $w_{P}$ (or by $\left[6\right.$, Lemma 2.2]). Suppose that $\min _{i}\left\{w_{P}\left(l_{i} d_{i}\right)\right\}=w_{P}\left(l_{k}\right)$. Then $w_{P}\left(l_{k}\right)=t$ for some $t \in \mathbb{Z}$, so if $\pi$ is a uniformizer for $\mathcal{O}_{P}$, then $w_{P}\left(\pi^{-t} l_{i}\right) \geq 0$ for all $i$, and $w_{P}\left(\pi^{-t} l_{k}\right)=0$. These facts hold since $\pi \in F=$ $Z(D)$. Thus, we may assume that $w_{P}\left(l_{i}\right) \geq 0$ for all $i$ and $w_{P}\left(l_{k}\right)=0$. Then $\overline{\sum_{i} l_{i} d_{i}}=\sum_{i} \overline{l_{i}} \overline{d_{i}} \neq 0$ since $\overline{l_{k}} \neq 0$, so $w_{P}\left(\sum_{i} l_{i} d_{i}\right)=0$, as desired.

For the second part, suppose that $P=P_{i}$ is one of the rational points at which the local index of $D$ is equal to $r=\operatorname{ind}(D)$. Let $\operatorname{Inv}\left(D_{P}\right)=k / r+\mathbb{Z}$ with $\operatorname{gcd}(k, r)=1$. Then $D_{P}=\left(l F_{P} / F_{P}, \sigma, \pi^{k}\right)$ for some uniformizer $\pi$ of $\mathcal{O}_{P}$. Since $x^{r}=\pi^{k} u$ for some unit $u \in \mathcal{O}_{P}^{*}$, and since $\left.w_{P}\right|_{F}=r v_{P}$ if $v_{P}$ is the normalized valuation on $F$ with valuation $\operatorname{ring} \mathcal{O}_{P}$, we see that $w_{P}\left(x^{i}\right)=i k$. Moreover, $L / F$ is unramified at $P$, so the value group of $L$ is $r \mathbb{Z}$. Thus, since $\operatorname{gcd}(r, k)=1$, the values $w_{P}\left(x^{i}\right)$ are distinct modulo the value group of $L$. If $\sum_{i} l_{i} x^{i} \in D$, each term $l_{i} x^{i}$ has distinct value, so $w_{P}\left(\sum_{i} l_{i} x^{i}\right)=\min _{i} w_{P}\left(l_{i} x^{i}\right)=\min _{i}\left\{w_{P}\left(l_{i}\right)+w_{P}\left(x^{i}\right)\right\}$; the latter equality holds since $w_{P}$ is a valuation on $D$. This shows that $1, x, \ldots, x^{r-1}$ is a strongly orthogonal basis of $D$ over $L$ with respect to $\Lambda_{P}$.

Proposition 9 The set $\left\{1, x, \ldots, x^{r-1}\right\}$ is an orthogonal basis with respect to each $\Lambda_{P}$ for $P \in X$.

Proof: Let $P \in X$. If $P=P_{i}$, then the $x^{i}$ form a strongly orthogonal basis with respect to $\Lambda_{P}$ by Lemma 8 . Next, suppose that $D$ is split at $P$. Recall from the proof of Lemma 7 that $l \mathcal{O}_{P}$ is the integral closure of $\mathcal{O}_{P}$ in $L$. From this, we see that $B=l \mathcal{O}_{P}$ is contained in the stalk $\Lambda_{P}$. By $[4$, Prop. 1.3], there are $z_{i} \in \Lambda_{P}$ such that $\Lambda_{P}=\oplus_{i=0}^{r-1} B z_{i}$ with $z_{i} b=\sigma^{i}(b) z_{i}$ for $b \in L$, and $z_{i} z_{j}=f\left(\sigma^{i}, \sigma^{j}\right) z_{i+j}$ for some normalized cocycle $f$ with values in $B$. Since $D$ is split at $P$, the maximal order $\Lambda_{P}$ is Azumaya over $\mathcal{O}_{P}$. Thus, $J\left(\Lambda_{P}\right)=\mathfrak{m}_{P} \Lambda_{P}=\oplus_{i=0}^{r-1} \mathfrak{m}_{P} B z_{i}$, where $\mathfrak{m}_{P}$ is the maximal ideal of $\mathcal{O}_{P}$. However, by [4, Prop. 3.1], $J\left(\Lambda_{P}\right)=\oplus_{i=0}^{r-1} I_{i} z_{i}$, where $I_{i}$ is the product of the maximal ideals $M$ of $B$ for which $f\left(\sigma^{i}, \sigma^{-i}\right) \notin M$. Since $\mathfrak{m}_{P} B$ is the product of all the maximal ideals of $B$, unique factorization of ideals in $B$ forces $f\left(\sigma^{i}, \sigma^{-i}\right) \notin M$ for every maximal ideal $M$ of $B$, so $f\left(\sigma^{i}, \sigma^{-i}\right) \in B^{*}$ for each $i$. Then, from the cocycle condition, we see that $\sigma^{i}\left(f\left(\sigma^{-i}, \sigma^{j}\right)\right) f\left(\sigma^{i}, \sigma^{-i+j}\right)=f\left(\sigma^{i}, \sigma^{-i}\right) f\left(1, \sigma^{j}\right) \in B^{*}$, so all cocycle values are in fact in $B^{*}$. Consequently, the residue $\operatorname{ring} \bar{A}$ is equal to the crossed product $\left(\bar{B} / \overline{\mathcal{O}_{P}}, \sigma, \bar{f}\right)=\oplus_{i=0}^{r-1} \bar{B} \overline{z_{i}}$, so the $\overline{z_{i}}$ are linearly independent over $\bar{B}$. Therefore, the $z_{i}$ form a strongly orthogonal basis with respect to $\Lambda_{P}$ by 
Lemma 8. Note also that $w_{P}\left(z_{i}\right)=0$ for each $i$ from this description of the residue ring $\bar{A}$. To see that the powers of $x$ form an orthogonal basis with respect to $\Lambda_{P}$, write $x^{i}=\alpha_{i} z_{i}$ with $\alpha_{i} \in L$. We have

$$
w_{P}\left(\sum_{i} l_{i} x^{i}\right)=w_{P}\left(\sum_{i} l_{i} \alpha_{i} z_{i}\right)=\min _{i}\left\{w_{P}\left(l_{i} \alpha_{i} z_{i}\right)\right\}=\min _{i}\left\{w_{P}\left(l_{i} x^{i}\right)\right\},
$$

since $z_{0}, \ldots, z_{r-1}$ is an orthogonal basis of $D$ with respect to $\Lambda_{P}$. Thus, the $x^{i}$ form an orthogonal basis.

Theorem 10 If $G$ is a divisor on $D$ with $\operatorname{deg}(G)<r n$ and $\operatorname{supp}(G) \cap$ $\left\{\Lambda_{P_{1}}, \ldots, \Lambda_{P_{n}}\right\}=\varnothing$, then $\mathcal{L}(G) \subseteq L$.

Proof: Let $f=\sum_{i} l_{i} x^{i} \in \mathcal{L}(G)$, so $w_{Q}(f)+w_{Q}(G) \geq 0$ for all $Q$. However, $w_{Q}(f)=\min _{i}\left\{w_{Q}\left(l_{i} x^{i}\right)\right\} \leq w_{Q}\left(l_{0}\right)$ by Proposition 9 , so $l_{0} \in \mathcal{L}(G)$. Let $f^{\prime}=\sum_{i=1}^{r-1} l_{i} x^{i}=f-l_{0} \in \mathcal{L}(G)$. Then $w_{P_{j}}\left(f^{\prime}\right) \geq 0$ for all $j$. Since $w_{P_{j}}\left(f^{\prime}\right)=$ $\min _{i>0}\left\{w_{P_{j}}\left(l_{i}\right)+i w_{P_{j}}(x)\right\}$, again by Proposition 9 , each $w_{P_{j}}\left(l_{i}\right)+i w_{P_{j}}(x) \geq 0$. But $i w_{P_{j}}(x) \neq 0$ in $\Gamma_{\Lambda_{P_{j}}} / \Gamma_{\Lambda_{P_{j}} \cap L}$ for each $i \neq 0$, so in fact $w_{P_{j}}\left(l_{i}\right)+i w_{P_{j}}(x)>0$ for each $i$. Consequently, $w_{P_{j}}\left(f^{\prime}\right)>0$ for each $j$. The coefficient of $\Lambda_{P_{j}}$ in $\left(f^{\prime}\right)+G$ is then at least 1 . The degree of $\Lambda_{P_{j}}$ is $r$ since the residue ring of $\Lambda_{P_{j}}$ is $l$. This, together with $\left(f^{\prime}\right)+G \geq 0$, forces $\operatorname{deg}\left(\left(f^{\prime}\right)+G\right) \geq r n$, while $\operatorname{deg}\left(\left(f^{\prime}\right)+G\right)=\operatorname{deg}\left(\left(f^{\prime}\right)\right)+\operatorname{deg}(G) \leq \operatorname{deg}(G)<r n$, a contradiction unless $f^{\prime}=0$. Therefore, $f=l_{0} \in L$.

Corollary 11 Let $G$ be a divisor on $D$ with $\operatorname{supp}(G) \cap\left\{\Lambda_{P_{1}}, \ldots, \Lambda_{P_{n}}\right\}=\varnothing$, and suppose that $\operatorname{deg}(G)<r n=\operatorname{deg}(\mathcal{P})$. If $\psi$ is the natural map $\psi$ : $\operatorname{div}(F) \rightarrow \operatorname{div}(L)$, then the code $C_{D}(\mathcal{P}, G)$ is equal to the code $C_{L}(\psi(\mathcal{P}), \psi(G))$.

Proof: We have seen in Lemma 7 that $C_{L}(\psi(\mathcal{P}), \psi(G))$ is equal to the subcode $\operatorname{ev}(\mathcal{L}(G) \cap L)$ of $C_{D}(\mathcal{P}, G)$. Since $\mathcal{L}(G) \subseteq L$, this image is equal to $C_{D}(\mathcal{P}, G)$

\section{References}

[1] S. Chase, D. Harrison, A. Rosenberg, Galois theory and cohomology of commutative rings, Mem. Amer. Math. Soc. 52, (1965), 1-19.

[2] E. Formanek, The Polynomial Identities and Invariants of $n \times n$ Matrices, CBMS Notes, Vol. 78, Amer. Math. Soc. Providence, 1991. 
[3] V. D. Goppa, Codes on algebraic curves, Soviet Math. Dokl., 24 (1981), $170-172$.

[4] D. Haile, Crossed-product orders over discrete valuation rings, J. Algebra 105 (1987), 116-148.

[5] I. Reiner, Maximal Orders, Academic Press, London, 1975.

[6] P. Morandi, Value functions on central simple algebras, Trans. Amer. Math. Soc. 315, 1989, 605-622.

[7] P. Morandi, Noncommutative Prüfer rings, J. Algebra 161, (1993), 324341.

[8] M. Orzech and C. Small, The Brauer Group of Commutative Rings, Marcel Dekker, Inc., New York, 1975.

[9] M. Van den Bergh and J. Van Geel, A duality theorem for orders in central simple algebras over function fields, J. Pure Appl. Algebra 31 (1984), 227-239.

[10] M. Van den Bergh and J. Van Geel, Algebraic elements in division algebras over function fields of curves, Israel J. Math. 52 (1985), 33-45.

[11] J. Van Geel, Places and Valuations in Noncommutative Ring Theory, Marcel Dekker, Inc., New York, 1981.

[12] E. Witt, Riemann-Rochster satz und $Z$-funcktion im hyperkomplexen, Math. Ann. 110 (1934), 12-28. 